$\begin{array}{cl}\begin{array}{c}\text { Revue } \\ \text { de Ihistoire }\end{array} & \text { Revue de l'histoire des religions } \\ \text { des religions } & \begin{array}{l}2 \mid 2012 \\ \text { Élection, Loi et communauté : de l'Alliance à la cité } \\ \text { chrétienne }\end{array}\end{array}$

\title{
Institutionnalisation de la grâce et retour de la Loi : un parcours médiéval
}

The Institutionalization of Grace and the Return of the Law: A Medieval Journey

Dominique logna-Prat

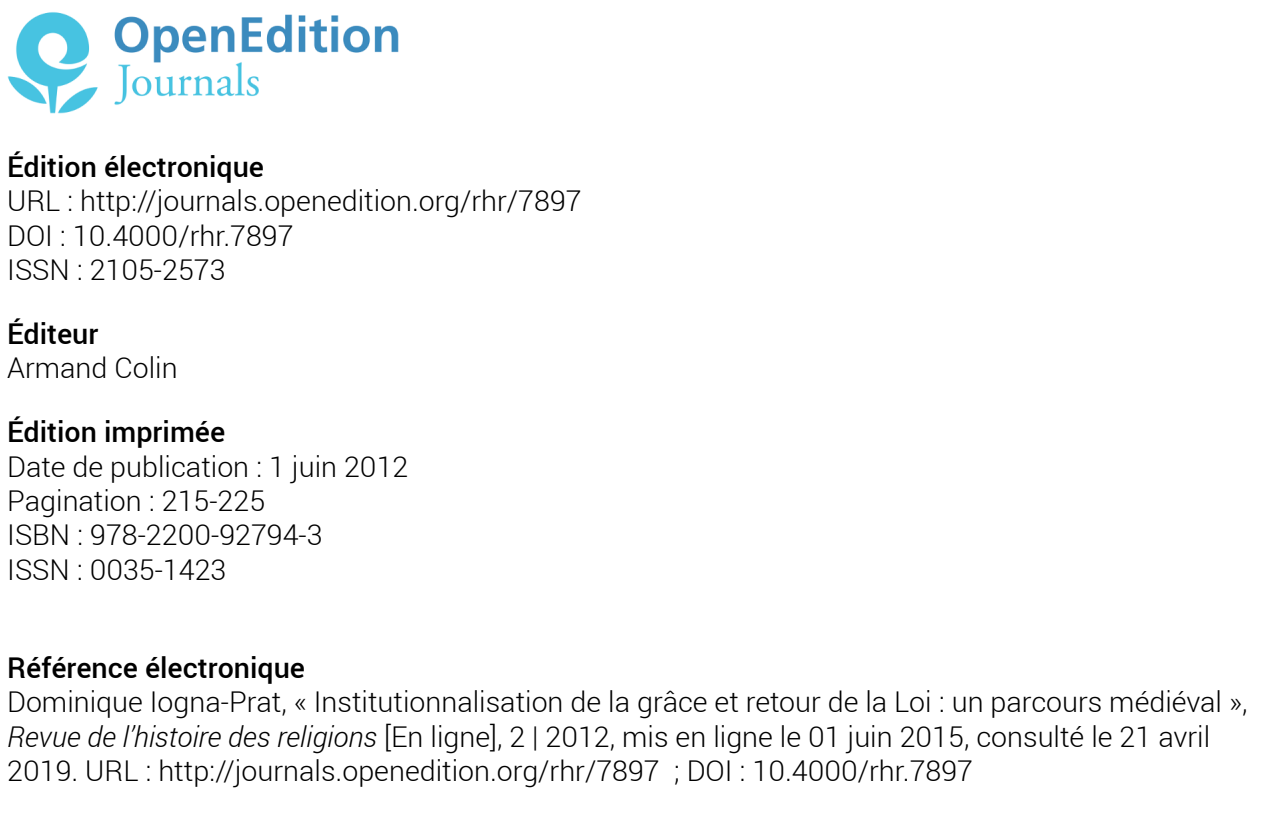

Tous droits réservés 


\section{Institutionnalisation de la grâce et retour de la Loi : un parcours médiéval}

Sous ce titre un peu énigmatique, l'article s'emploie à commenter Solitude de l'incomparable de Milad Doueihi en traitant du christianisme sur son versant institutionnel. En plaçant résolument au centre de la discussion le Moyen Âge, période qu'enjambe le parcours philosophique d'Augustin à Spinoza que propose l'auteur, il s'agit de comprendre comment, avant Spinoza, s'est opéré le découplage théologie/politique à la genèse de la pensée politique et des pratiques sociales de l'Occident moderne. Le parcours institutionnel esquissé aborde trois questions principales: la prédestination, la dimension économique de la grâce, et l'universalisme chrétien.

\section{The Institutionalization of Grace and the Return of the Law : A Medieval Journey}

This article, with its rather enigmatic title, attempts to commentate Milad Doueihi's Solitude of the Incomparable by dealing with the institutional side of Christianity. Placing the Middle Ages-a period that is spanned by the philosophical journey leading from Augustine to Spinoza to which the title refers-firmly in the center of the discussion aids in an understanding of how, before Spinoza, theology and politics were decoupled at the birth of political thought and of the social practices of the modern West. The institutional trajectory that is sketched here addresses three main questions: predestination, the economic dimension of grace and Christian universalism. 
«Institutionnalisation de la grâce et retour de la Loi : un parcours médiéval»: ce titre un peu énigmatique et sans doute un peu trop emphatique ne vise en fait qu'à fournir quelques annotations en commentaire à Solitude de l'incomparable ${ }^{1}$. Dans son essai, Milad Doueihi propose un parcours philosophique d'Augustin à Spinoza et à sa réception, sous la forme d'un enjambement classique du Moyen Âge qui n'est pas sans poser problème à l'historien du Moyen Âge que je suis, désireux de comprendre le découplage théologie/politique à la genèse du politique dans la pensée et les pratiques sociales de l'Occident moderne. La discussion ouverte autour de Solitude de l'incomparable offre une possibilité plutôt rare de surmonter la regrettable mise à l'écart de la médiévistique de tout questionnement partagé au sein des sciences sociales propre à dépasser le statut d'un Moyen Âge pur antonyme de la Modernité.

Avant de remettre l'histoire de l'Occident médiéval à une place un peu plus exposée, commençons par quelques questions de fond. Quelle valeur attribuer à la distinction élection/grâce, «conversion» de l'élection par la grâce «dans sa finalité historique et eschatologique avec passage du particulier à l'universel», avec l'avènement du corps collectif, de l'Ecclesia, de la Cité et du genre humain (p. 45)? Comment apprécier cette universalisation du christianisme par la tradition philosophique dans l'héritage de Spinoza, et la distinction radicale judaïsme/christianisme, à la manière de Hegel et de sa «scission infinie», ou à la manière de Kant, qui voyait dans le christianisme la seule vraie «religion», placée en surplomb des «croyances» et des «confessions», l'unique religion menant à l'éthique universelle? Faut-il insister, au contraire, en reprenant la distinction pascalienne entre «fausseté et vérité des religions», sur le legs commun des monothéismes? C'est l'option de Jan Assmann, dans Le prix du monothéisme, qui fait globalement des monothéismes des religions de l'élection, de la distinction du vrai et du faux ${ }^{2}$. Ce faisant, peut-on voir, dans le christianisme, un prolongement du judaïsme sur le mode de

1. Les chiffres entre parenthèses renvoient aux pages de l'ouvrage discuté.

2. Jan Assmann, Le prix du monothéisme, Paris, Aubier, 2007 [éd. orig., Munich-Vienne, 2003]. 
«l'universalité de soi», c'est-à-dire une autre forme de distinction entre «nous » et les «autres», une «universalité de soi» postulant la réduction des autres à «nous» comme seuls «élus» possibles? Ce serait remettre en selle, à l'horizon chrétien, la notion d' «élection», pas vraiment prise en considération par $\mathrm{M}$. Doueihi, alors qu'elle est d'usage courant tout au long de l'histoire chrétienne, sous les formes multiples des «signes de l'élection».

De ce point de vue, la discussion sur l' «élite du monde» menée par M. Doueihi en référence au Kuzari de Juda Hallévi me semble devoir être prolongée. Selon Hallévi, l'élection est un wacqf, un legs inaliénable qui fait la communauté, qui constitue la nation, comme un corps, une voix, un territoire uniques, sans apport extérieur, sans assimilation, sans conversion possibles; en ce sens, M. Doueihi oppose le «peuple élu» à l'Église comme corps mystique, et à l'Oummah, comme nation de la dernière prophétie (p. 141). Mais que dire de l'exclusivisme chrétien, contemporain de la mise en place d'une «société de persécution», autour des années 1100, par définition stricte de la société chrétienne comme communauté sacramentelle distinguant radicalement les chrétiens et les autres ${ }^{3}$ ? Que dire de la définition généalogique de l'appartenance chrétienne, avec un rapport sacral à la «terre chrétienne» qui engendre les fidèles - une définition quasi raciale du chrétien, avec l'émergence du thème de l'impossible conversion et de la «pureté du sang », dont on sait la profondeur dans l'histoire de l'idée de race en Occident depuis le $\mathrm{XV}^{\mathrm{e}}$ siècle au plus tard ${ }^{4}$ ?

On aura compris que, dans mon commentaire de Solitude de l'incomparable, je propose une orientation de lecture visant à remettre en place l'institution ecclésiale, que l'approche par la seule patristique ne permet pas vraiment d'envisager, l'Église comme «institution totale», porteuse exclusive d'une définition du social,

3. Robert I. Moore, La persécution. Sa formation en Europe $X^{e}$-XIII ${ }^{e}$ siècle, Paris, Les Belles Lettres, 1991 [éd. orig., Oxford, 1987].

4. Yosef H. Yerushalmi, «L'antisémitisme racial est-il apparu au $\mathrm{xx}^{\mathrm{e}}$ siècle? De la limpieza de sangre espagnole au nazisme», Esprit 1990 (1993), p. 5-35. Sur la question afférente du métissage, dans une bibliographie surabondante on renverra à une commode introduction historique et à une remarquable étude de cas: Carmen Bernand, Serge Gruzinski, Histoire du Nouveau Monde, 2: les métissages, Paris, Fayard, 1993; Anne Levallois, Les écrits autobiographiques d'Ismayl Urbain, homme de couleur, saint-simonien et musulman (1812-1884), Paris, Maisonneuve \& Larose, 2005. 
étant une réalité médiévale que nous avons tout intérêt à rappeler si nous voulons sortir d'une histoire de la pensée référée à quelques grands noms flottant dans le temps. L'enjeu de cette remise en place de l'institution ecclésiale médiévale est de permettre d'aborder la question des conditions historiques d'émergence du spinozisme, en amont d'une histoire des doctrines philosophico-politiques faisant une part trop exclusive au tournant de la «Modernité»; d'éclairer la longue genèse médiévale, dans un incessant va-et-vient entre discours et pratiques institutionnelles du gouvernement des hommes, du découplage entre sphères du «théologique» et du «politique».

\section{UN PREMIER TOURNANT INSTITUTIONNEL：LA PRÉDESTINATION?}

De façon surprenante, Solitude de l'incomparable, ce livre qui embrasse aussi généreusement et aussi largement la question de l'élection et de la grâce, n'aborde pas le problème de la prédestination. Je m'y arrête rapidement pour commencer, afin de marquer la dimension institutionnelle quelque peu paradoxale du problème. Mais le «paradoxe» est d'une très grande richesse pour mieux comprendre l'histoire à venir ${ }^{5}$.

La doctrine d'Augustin, appelée à être rediscutée au cours du Moyen Âge, spécialement à l'époque carolingienne, tient en quatre points principaux: la faute d'Adam a condamné tous les hommes; seul un petit nombre doit être sauvé; le salut se fait par élection divine, gratuite, sans mérite propre; le reste des hommes est placé hors du salut pour des raisons que l'on ne connaît pas. La doctrine d'Augustin pose le problème de la liberté humaine. La grâce divine permet-elle aux hommes d'agir au moins un peu sur le destin qui leur a été individuellement assigné par le Créateur? La communauté peut-elle contribuer au salut? La première question est au centre de la querelle dite pélagienne et du volontarisme ascétique qui traverse toute l'histoire du monachisme au Moyen Âge et des différentes formes de conception de la voie courte des mystiques. La seconde pose tout le problème de la prise en charge des fidèles par la communauté à une époque qui, avec le baptême des enfants

5. Sur une question aussi complexe, étudiée depuis des générations, impossible de faire le moindre renvoi, même à une bibliographie de base. Je m'en tiendrai donc à de rares et nécessaires indications concernant le Moyen Âge. 
et l'invention du parrainage, accorde une place déterminante à «la foi pour l'autre» et à la circulation de la caritas au sein du grand corps qu'est l'Église'.

On commence à entrevoir, ce faisant, dans quelle mesure la question de la prédestination a pu s'institutionnaliser. Un tournant essentiel en ce sens me semble s'accomplir à l'époque carolingienne quand les moines, fonctionnellement voués au salut collectif dans l'isolement du cloître, lient explicitement la question de la prédestination à la pénitence. Haymon d'Auxerre ( $f$. 820-840), par exemple, soutient que le choix de la pénitence volontaire dans le retrait du monde est une preuve d' «élection», potentiellement ouverte à tous les fidèles? ${ }^{7}$. La grâce gratuitement offerte par Dieu consiste ainsi dans le libre choix de pratiquer la pénitence, propédeutique anticipatrice de l'intégration dans la communauté des saints, le monastère, véritable concentré d'Église, marquant en quelque sorte l'entrée dans l'espace du salut - une entrée fonctionnelle qui n'a de sens que dans le cadre d'une répartition des tâches globale au sein de la société chrétienne conçue selon le fameux schéma trifonctionnel (prier, combattre, produire) ${ }^{8}$.

La question du «cadre de l'élection», si je puis dire, rebondit quelques siècles plus tard sous l'influence d'Aristote - l'Aristote de la Politique et de l'Éthique à Nicomaque, que l'on redécouvre et commente d'abondance dans l'Occident latin à partir des années $1250^{\circ}$. Je me limite à un exemple pour illustrer l'importance

6. Présentation générale du problème avec renvoi aux principaux travaux de référence dans Dominique Iogna-Prat, Ordonner et exclure. Cluny et la société chrétienne face à l'hérésie, au judaïsme et à l'islam (100-1150), Paris, Aubier, 1998, chap. 5 et 7 .

7. Alfonso Hernandez, Anthropologie et ecclésiologie dans l'exégèse biblique carolingienne selon le Commentaire sur le prophète Osée d'Haymon d'Auxerre, thèse en co-tutelle, Universidad de Buenos Aires et Université de Paris 1 Panthéon Sorbonne, 2009; résumé dans le Bulletin du Centre d'études médiévales d'Auxerre, 14 (2010): http//com.revues.org/index11596.html

8. Edmond Ortigues, «Haymon d'Auxerre, théoricien des trois ordres», dans L'École carolingienne d'Auxerre, de Murethac à Remi, 830-908, D. Iogna-Prat, Colette Jeudy, Guy Lobrichon (dir.), Paris, Beauchesne, 1991, p. 181-227 [repris dans Id., La révélation et le droit, Paris, Beauchesne, 2007].

9. Catherine König-Pralong, Avènement de l'aristotélisme en terre chrétienne, Paris, Vrin, 2005. Pour une approche globale de la réception de l'Aristote «politique»: Christoph Flüeler, Rezeption und Interpretation der Aristotelischen Politica im späten Mittelalter, 2 vol., Amsterdam/Philadelphia, 1992. 
que prend alors la polis, en recouvrant, en doublant l'Église, comme «contenu» nécessaire à la réception de la grâce divine. $\mathrm{Au}$ douzième livre de sa somme théologico-morale intitulée $L e$ chrétien (qui est généralement considérée comme la dernière des grandes sommes scolastiques), le franciscain catalan Francesc Eiximenis (1330-1409) traite longuement de la chose publique et du gouvernement des hommes dans le seul cadre social possible pour lui et ses contemporains: la ville. Il commence par traiter de l'homme intérieur et de la façon dont le Souverain Créateur a, aux commencements du monde, «gravé la Cité du Paradis en l'homme». Et, en référence explicite à Augustin - mais un Augustin revisité à la lecture d'Aristote et des discussions de Thomas d'Aquin sur les deux «ordres» (ordre naturel et ordre de la grâce) - d'enchaîner sur le fait que Dieu donne à l'homme l'inclination à vivre en communauté :

«Le souverain gouverneur et père, Dieu Notre-Seigneur [...] donna [à l'homme] une inclination naturelle à vivre en compagnie, une compagnie réglée et dirigée selon l'esprit et le corps, la cité matérielle. Le but est que l'homme, en prenant soin de cette cité matérielle, connaisse la cité spirituelle qu'il porte en soi. Dans l'observation de ces deux cités, de leur beauté, de leur noblesse, il désire la cité souveraine et céleste. Il accomplit les œuvres qui lui permettent de la mériter et de la posséder. $»^{10}$

\section{L'ÉCONOMIE DU CHRISTIANISME}

En référence à Augustin, M. Doueihi fait d'utiles rappels sur la dimension économique de la grâce, qui amène le fidèle à «rendre», entrer, participer à «un commerce du mutuel échange». Sur la base d'une formule d'Augustin («nous sommes tous la monnaie de Dieu»), il insiste à juste titre sur l'importance des métaphores monétaires et économiques dans la théologie chrétienne, ce qu'ont amplement démontré les travaux de Giacomo Todeschini sur le développement précoce d'un langage théologico-économique, puis sur l'émergence, à l'époque scolastique, d'une véritable pensée

10. Francesc Eiximenis, Le chrétien, trad. P. Gifreu, Perpignan, Éditions de La Merci, 2010, p. 235. Analyse globale de l'œuvre: Jean-Pierre Barraqué, «Les idées politiques de Francesc Eiximenis », Le Moyen Âge, 114 (2009/3-4), p. 531556. 
«économique» du «marché» chrétien ${ }^{11}$. M. Doueihi note tout aussi justement les implications sociologiques de la grâce, l'engagement de mutuelle bonne foi dans l'échange étant créateur «d'une nouvelle communauté où le jeu du don et de la dette gouverne le fonctionnement et l'organisation du social aussi bien que celle du spirituel» (p. 81). Mais il me semble nécessaire d'aller plus loin ${ }^{12}$.

Au sens ancien, grec, du terme, l'économie (oikonomia) est ce qui fixe les «règles» (nomoi) de bonne gestion de la «maison» (oikos), la Cité n'étant qu'une extension de la maison. Chez saint Paul, principal acteur de l'hellénisation du christianisme, «économie» est le terme qui s'impose pour parler du salut des chrétiens. L'oikonomia, c'est l'économie du Plérôme (la Plénitude); la saisie de la divinité dans la plénitude de sa perfection (Ephes. 1,10) relève de «l'économie de la grâce» $(3,2)$, de «l'économie du mystère» $(3,9)$, en un mot de «l'économie de Dieu» (Col. 1, 25). Comme l'a montré Giorgio Agamben, la distinction entre «l'être de Dieu» et ses «activités» relève de sphères intimement articulées - la théologie et l'économie -, lesquelles permettent de rendre compte de la «triple oikonomia» d'un Dieu trinitaire qui n'en reste pas moins, sur le plan ontologique, une puissance monadique ${ }^{13}$. C'est la logique de l'accomplissement du plan de Dieu, en quelque sorte la «glorification» de son «règne», qui justifie l'existence de l'Église, laquelle n'est rien d'autre que l'économie de Dieu, l'espace de ses manifestations, l'échelle médiatique permettant de remonter à l'Un et de s'y absorber.

Les Pères grecs et latins ont connaissance des sens anciens du terme économie, comme pratique et science de la gestion, de l'administration des biens et des personnes, dans le cadre de la maison et de la Cité, ce qui leur permet de mettre en place, dès les

11. En dernier lieu : Giacomo Todeschini, Richesse franciscaine. De la pauvreté volontaire à la société de marché, Paris, 2008 [éd. orig., Bologne, 2004]; sur cette œuvre importante: Valentina Toneatto, «La richesse des franciscains. Autour du débat sur les rapports entre économie et religion au Moyen Âge», Médiévales, 60, 2011, p. 187-202.

12. Pour plus de détails : D. Iogna-Prat, «Préparer l'au-delà, gérer l'ici-bas: les élites ecclésiastiques, la richesse et l'économie du christianisme (perspectives de travail)», dans Les élites et la richesse au haut Moyen Âge, Jean-Pierre Devroey, Laurent Feller, Régine Le Jan (éds.), Turnhout, Brepols, 2011, p. 59-70, dont quelques développements sont repris ici.

13. Giorgio Agamben, Le règne et la gloire, Paris, Le Seuil, 2008 [éd. orig., Vicenza, 2007] 
$\mathrm{II}^{\mathrm{e}}$ et $\mathrm{III}^{\mathrm{e}}$ siècles, une «économie ecclésiastique» avec la définition d'un ensemble de charges et d'offices au sein de l'institution ecclésiale naissante. Ils intègrent et développent également la notion d'économie paulinienne dans deux directions principales. La première relève de l'économie de l'incarnation (Rom. 2, 29; Cor. 3,6 ), suivant laquelle le Christ est l'oikonomia du Père. Le second apport des Pères consiste en une adaptation de la gestion humaine au plan providentiel, des moyens économiques aux fins du salut sous deux formes principales: l'identification du corps du Christ au corps de l'Église (I Cor. 12, 31); la définition de l'institution ecclésiale comme oikonomos de l'accomplissement, avec glissement de l'oikos domestique à l'oikos cosmologique. En ce sens, l'Église est dispensatrice de Dieu et du divin, qui sont en quelque sorte placés dans sa gestion. Elle est la visibilité de Dieu, sa nécessaire "glorification»; d'où l'importance de la notion «d'économie iconique» si bien mise en valeur par Marie-José Mondzain, l'enjeu de cette économie des images comme de toute manifestation matérielle, étant l'administration, la gestion des visibilités divines ${ }^{14}$. Ce problème capital dans l'évolution du christianisme au cours du Moyen Âge suppose d'être replacé dans le cadre d'ensemble de la discussion sur la notion de «médiation(s)» (médiations iconiques, sacramentelles, institutionnelles), l'économie étant «médiatrice» dans la mesure où elle permet non seulement la manifestation matérielle du divin, mais aussi une remontée vers l'au-delà par la transformation des biens terrestres. Tel est le rôle que joue l'œuvre du Pseudo-Denys l'Aréopagite (découverte dans l'Occident latin au tournant des années 800), qui offre à la fois le concept («hiérarchie»), la dynamique («procession») et le cadre (l'emboîtement des étages hiérarchiques trines) propres non seulement à penser le destin de la communauté chrétienne dans son aspiration vers l'Un mais aussi celui de chaque fidèle, en conjoignant de la sorte une ecclésiologie et une mystique. L'homologie posée entre «hiérarchie», élévation intérieure et hiérarchies sociales et cosmologiques (ou hiérarchies ecclésiastiques et célestes) pose qu'il ne saurait y avoir d'espace de la grâce en dehors d'une structure commune qui est l'Église. Telle est la «loi de hiérarchie» abondamment commentée tout au long du

14. Marie-José Mondzain, Image, icône, économie. Les sources byzantines de l'imaginaire contemporain, Paris, Le Seuil, 1998. 
Moyen Âge, une loi qui, en quelque sorte, institutionnalise la grâce, qui «institutionnalise» dans la mesure où elle fait des médiations un principe cosmologique, la reductio ad ima supposant de passer de l'inférieur au supérieur par le degré moyen.

\section{L'UNIVERSALISME CHRÉTIEN}

Classiquement, M. Doueihi reprend la longue marche occidentale vers l'autonomie et l'universalité, depuis Augustin jusqu'à la philosophie classique (Kant, Hegel), en passant par Spinoza. Je propose de faire retour à la question historique de l'universalisme chrétien, par un autre biais, plus institutionnel, en nous penchant rapidement sur l'évolution lexicale du terme «chrétienté».

\section{La Chrétienté}

La christianitas désigne, à l'origine, tout ce qui touche à l'identité des disciples du Christ par opposition au monde environnant voué à la paganitas, à la gentilitas; l'accaparement progressif de la sphère du sacré public par le christianisme déclaré religion d'État à partir de la fin du IV siècle introduit même l'opposition du «sacré chrétien» à la profanitas. C'est par assimilation de la christianitas au cadre politique et culturel de la romanitas que peut se développer le premier universalisme chrétien. Mais à haute époque, il ne s'agit pas simplement de l'aspiration d'une religion déjà prosélyte, qui a vocation à remplir tout l'espace de la Terre. Dans le fil de la lutte contre les Donatistes, qui refusent toute confusion entre la communauté chrétienne et la chose publique romaine, Augustin forge une intéressante formule de la «véritable» appartenance chrétienne par rapport aux dissidents en parlant de christianitas orbis terrae, en d'autres termes: la chrétienté de tous les fidèles catholiques par rapport à ceux qui dévient, l'Église universelle étant conçue comme la communio orbis terrarum ${ }^{15}$.

Le tournant essentiel dans l'universalisation de la notion de christianitas se produit entre 800 et 1100 , au moment où l'Église, en tant que structure hiérarchique englobante, s'impose comme

15. Augustin, Ep. 43, 7, 19. 
une monarchie spirituelle porteuse de la société chrétienne ${ }^{16}$. On passe alors de la «chrétienté», en tant que groupe d'appartenance différencié de son environnement (voire en rupture avec lui), à la «Chrétienté», espace d'inscription géopolitique exclusif de l'appartenance, qui, d'une certaine manière, se «naturalise»- le prosélytisme chrétien contemporain de la grande Réforme de l'Église des $\mathrm{XI}^{\mathrm{e}}-\mathrm{XII}^{\mathrm{e}}$ siècles et des premières croisades confondant désormais la Chrétienté (ou la société chrétienne) avec l'humanité, ce qui implique à la fois une inscription territoriale et une esquisse de définition raciale, qui, à l'aune de l'histoire occidentale, pèsera lourd dans la constitution, entre autres, d'une véritable judéophobie porteuse à terme d'antisémitisme.

\section{Théologie et politique}

Une fois qu'on a compris comment la première universalité chrétienne contemporaine d'Augustin évolue vers l'universalisme institutionnel de l'Église médiévale, tout le problème est d'expliquer comment a pu se faire le renversement qui aboutit à l'universalité éthique revendiquée par la tradition philosophique moderne. Où situer le tournant? Au carrefour que représente l'œuvre de Spinoza, comme le propose M. Doueihi? À mon sens plus tôt, avec le découplage entre le théologique et le politique contemporain de la scolastique.

Revenant à nouveaux frais sur le tournant fondateur de «l'esprit laïque» cher à Georges de Lagarde, le sociologue Louis Dumont a mis l'accent sur les effets à long terme du nominalisme et, tout spécialement, de l'œuvre de Guillaume d'Ockham (v. 1280-1349), qui marquerait le passage de l'uniuersitas médiévale à la societas moderne ${ }^{17}$. Ockham est amené à prendre ses distances par rapport à la double référence traditionnelle entre, d'une part, l'homme comme tout vivant, individu privé en relation directe avec son créateur et, d'autre part, l'homme membre de la communauté, partie du corps social. À Thomas d'Aquin, qui différenciait des «substances premières» (les

16. Dans l'attente de la thèse de doctorat en cours de Tim Geelart (Francfortsur-le-Main) sur la sémantique de christianitas au haut Moyen Âge: Régine Azria, Danièle Hervieu-Léger, dir., Dictionnaire des faits religieux, Paris, 2010, s.v. «Chrétienté» (Jérôme Baschet, D. Iogna-Prat).

17. Louis Dumont, Essaissurl'individualisme. Uneperspectiveanthropologique sur l'idéologie moderne, Paris, 1991 («Points»), p. 84-89. 
êtres particuliers, Pierre ou Paul; la «pétrité» de Pierre ou la «paulité» de Paul) et des «substances secondes» (genres, espèces, catégories, classes d'êtres), Ockham et le nominalisme opposent qu'il n'existe pas de «substances secondes» mais un simple phénomène de réification, c'est-à-dire l'emploi de termes généraux et arbitraires qui trouvent leur fondement et leur raison d'être dans la réalité empirique et qui ne renvoient qu'à eux-mêmes. Cette prise de position marquerait la naissance de l'individualisme dans la philosophie et dans le droit, car elle pose qu'il n'y a rien d'ontologiquement réel au-delà de l'être particulier (ou substance première); ce faisant, Ockham étendrait la liberté de l'individu, traditionnelle dans le christianisme, du plan de la vie personnelle à celui de la vie en société. Ce tournant marquerait le passage du religieux (l'Église comme Tout de la société) au politique (l'État comme Tout social) $^{18}$.

Comme l'a noté Sylvain Piron à l'heure de donner «congé à (Michel) Villey », on a sans doute tort de faire d'Ockham « un Hobbes par anticipation», et de lui attribuer l'exclusivité d'un moment théologico-politique en fait plus diversifié dans ses sources ${ }^{19}$. Pour autant, à défaut d'un acteur unique (Ockham), il convient d'insister sur l'importance d'un temps de la pensée, qu'on peut situer entre 1250 et 1350 , d'une séparation des sphères, d'un premier découplage du théologique et du politique préparant largement l'autonomie et le subjectivisme à l'œuvre dans les écrits de Spinoza, l'insistance mise à parler de la «réalité» de l'être particulier et du caractère «fictionnel», relatif, des cadres d'appartenance revenant à introduire la «pluralité» dans toute réflexion sur la vie des groupes, et à libérer, au moins potentiellement, l'universel pour un investissement éthique. On est loin, on le voit, d'un long fleuve tranquille d'Augustin à Spinoza: puisse cette discussion autour du beau livre de M. Doueihi nous en persuader.

d.iogna-prat@wanadoo.fr

18. Arthur S. McGrade, The Political Thought of William of Ockham: Personal and Institutionnal Principles, Cambridge, 1974.

19. Sylvain Piron, «Congé à Villey», dans Historiographie de la pensée politique médiévale, Atelier du Centre de recherches historiques, 1 (2008), http:// acrh.revues.org/index314.html 